\title{
Intercostal and forearm muscle deoxygenation during respiratory fatigue in patients with heart failure: potential role of a respiratory muscle metaboreflex
}

\author{
A.M. Moreno ${ }^{1,3}$, R.R.T. Castro ${ }^{3}$, B.M. Silva $^{2,3}$, H. Villacorta $^{3}$, \\ M. Sant'Anna Junior ${ }^{1}$ and A.C.L. Nóbrega ${ }^{2,3}$ \\ ${ }^{1}$ Procordis Hospital Cardiológico, Niterói, RJ, Brasil \\ ${ }^{2}$ Departamento de Fisiologia e Farmacologia, Universidade Federal Fluminense, Niterói, RJ, Brasil \\ ${ }^{3}$ Programa de Pós-graduação em Ciências Cardiovasculares, Universidade Federal Fluminense, Niterói, RJ, Brasil
}

\begin{abstract}
The purpose of this study was to determine the effect of respiratory muscle fatigue on intercostal and forearm muscle perfusion and oxygenation in patients with heart failure. Five clinically stable heart failure patients with respiratory muscle weakness (age, $66 \pm 12$ years; left ventricle ejection fraction, $34 \pm 3 \%$ ) and nine matched healthy controls underwent a respiratory muscle fatigue protocol, breathing against a fixed resistance at $60 \%$ of their maximal inspiratory pressure for as long as they could sustain the predetermined inspiratory pressure. Intercostal and forearm muscle blood volume and oxygenation were continuously monitored by near-infrared spectroscopy with transducers placed on the seventh left intercostal space and the left forearm. Data were compared by two-way ANOVA and Bonferroni correction. Respiratory fatigue occurred at $5.1 \pm 1.3 \mathrm{~min}$ in heart failure patients and at $9.3 \pm 1.4$ min in controls $(P<0.05)$, but perceived effort, changes in heart rate, and in systolic blood pressure were similar between groups $(P>0.05)$. Respiratory fatigue in heart failure reduced intercostal and forearm muscle blood volume $(\mathrm{P}<0.05)$ along with decreased tissue oxygenation both in intercostal (heart failure, $-2.6 \pm 1.6 \%$; controls, $+1.6 \pm 0.5 \% ; P<0.05$ ) and in forearm muscles (heart failure, $-4.5 \pm 0.5 \%$; controls, $+0.5 \pm 0.8 \%$; $P<0.05$ ). These results suggest that respiratory fatigue in patients with heart failure causes an oxygen demand/delivery mismatch in respiratory muscles, probably leading to a reflex reduction in peripheral limb muscle perfusion, featuring a respiratory metaboreflex.
\end{abstract}

Key words: Heart failure; Metaboreflex; Muscles respiratory

\section{Introduction}

Along with a limited ability to increase cardiac output, increased pulmonary capillary pressure and skeletal myopathy, respiratory muscle fatigue is considered a key factor contributing to the diminished exercise capacity observed in patients with heart failure $(\mathrm{HF})(1,2)$. The main mechanism linking respiratory muscle fatigue to reduced exercise capacity seems to depend on the activation of a respiratory muscle metaboreflex. The stimulation of ergoreceptors sensitive to accumulation of metabolites within respiratory skeletal muscle reflexively increases sympathetic outflow, which limits the increase of blood flow to working muscle, causing reduced perfusion of locomotor muscles and leading to fatigue (3-5). The unloading of respiratory muscle prevents respiratory fatigue, reducing reflex sympathetic activation and allowing for increased blood flow to locomotor muscles $(6,7)$. The proposed trigger for the metaboreflex is accumulation of metabolites within respiratory muscle caused by an oxygen demand/delivery mismatch $(3,8)$, but so far the patterns of respiratory blood flow and oxygenation during respiratory fatigue in patients with heart failure have not been described. Therefore, this study was designed to determine the effect of respiratory muscle fatigue on intercostal and forearm muscles blood volume and oxygenation in HF patients.

\section{Material and Methods}

\section{Patients}

Patients with HF were eligible to participate in this study if they were functional class II or III (New York Heart 
Association), had a left ventricular ejection fraction $<50 \%$, respiratory muscle weakness (defined as maximal inspiratory pressure $\leq 70 \%$ of predicted value), were clinically stable, and had had no change in medication in the previous 3 months. Patients with a history of primary pulmonary disease, expired volume in $1 \mathrm{~s} /$ forced vital capacity $<70 \%$, a smoking habit in the previous 3 months, diabetes mellitus, unstable angina, significant cardiac arrhythmias or myocardial infarction within the previous 6 months, or had previously participated in any cardiac rehabilitation program were excluded. All subjects abstained from heavy exercise and caffeine prior to testing. A group of age-matched healthy subjects was tested as controls. The study complied with the Declaration of Helsinki, the protocol was approved by the Institutional Ethics Committee of Universidade Federal Fluminense, and informed consent was obtained from all subjects. The required minimum sample size of 5 subjects per group was calculated for a 2sided test for independent samples, assuming a size effect of $4.3 \%$ and mean standard deviation of $2.3 \%$ for $\alpha=0.05$ and a power of 0.80 .

\section{Experimental protocol and measurements}

Maximal inspiratory pressure was measured with a pressure transducer (MVD-300 V.1.1 Microhard System, Globalmed, Brazil) connected to a system with two unidirectional valves (DHD Inspiratory Muscle Trainer, USA). Fatigue was induced by a protocol where the patients breathed for as long as they could against a resistance established at $60 \%$ of their maximal inspiratory pressure (Threshold Inspiratory Muscle Training, Heathscan Products Inc., USA), corresponding to an absolute inspiratory pressure of $23-42 \mathrm{cmH}_{2} \mathrm{O}$. Fatigue was indicated by inability of the patient to achieve at least $80 \%$ of the predetermined inspiratory pressure during three consecutive breaths. Intercostal and limb muscle blood volume and oxygenation were continuously recorded by near-infrared spectroscopy (NIRS; Oxiplex TS, ISS, USA) using transducers having a fixed $3-\mathrm{cm}$ distance between the light source and detector. The transducers were placed over the seventh left intercostal space as described by Vogiatzis et al. (9), between the anterior axillary line and the midclavicular line beyond the insertion of serratus anterior, and on the ventral surface of the left forearm. NIRS estimates local microvascular concentration of oxyhemoglobin + myoglobin (oxy- $\mathrm{Hb}$ ) and deoxyhemoglobin + myoglobin by measuring light absorbency at 850 and $760 \mathrm{~nm}$, respectively. Muscle blood volume was estimated by total hemoglobin/myoglobin and muscle oxygenation by the proportion of oxy- $\mathrm{Hb}$ to total $\mathrm{Hb}$. During the respiratory fatigue protocol, arterial oxygen saturation $\left(\mathrm{SpO}_{2}\right)$ was measured continuously by finger pulse oximetry (MorryaM1000, Brazil), and the perceived effort was quantified by the $0-10$ Borg scale. Body mass index (BMI) was calculated and defined as the ratio between the body weight in kilograms and the square of the height in meters.
Baseline values were considered as the mean of the last $3 \mathrm{~min}$ before the fatigue protocol started, and recovery was the mean of the first minute after completing the protocol. NIRS results are reported as the mean of the values obtained during the first, second, and third minutes of the protocol and the "fatigue" point was considered as the average of the last $3 \mathrm{~s}$ before fatigue occurred. Results are reported as means $\pm S D$, except for $\mathrm{SpO}_{2}$, which is reported as median and extreme values. Data were compared by two-way ANOVA followed by the Bonferroni post hoc test, where group (HF patients and healthy controls) and moment (baseline, each minute, fatigue, and recovery) were the main factors.

\section{Results}

Five clinically stable HF patients with respiratory muscle weakness were included (4 males and 1 female, $66 \pm 12$ years of age, BMI $27.6 \pm 0.6 \mathrm{~kg} / \mathrm{m}^{2}$, left ventricular ejection fraction $34 \pm 3 \%$ ). The mean maximal inspiratory pressure was $61 \pm 13 \%$ of the predicted value. The etiology was ischemic cardiomyopathy in three patients and nonischemic in two. All patients received the optimal recommended treatment for $\mathrm{HF}$, including renin-angiotensin system antagonists $(5 / 5)$, aldosterone antagonists $(5 / 5)$, beta-blockers $(4 / 5)$, diuretics $(3 / 5)$, hydralazine $(3 /$ $5)$, statins (5/5) and acetylsalicylic acid (4/5). Nine healthy subjects without respiratory muscle weakness $(6$ males, 3 females, $65 \pm 13$ years of age, BMI $27.4 \pm 0.7 \mathrm{~kg} / \mathrm{m}^{2}$, mean maximal inspiratory pressure $95 \pm 20 \%$ predicted value) were enrolled in the study as controls.

Patients with HF developed respiratory fatigue earlier $(5.1 \pm 1.3 \mathrm{~min})$ in the respiratory protocol than controls $(9.3 \pm 1.4 \mathrm{~min} ; \mathrm{P}<0.05)$. Figure 1 shows that during respiratory fatigue, changes in heart rate $(\mathrm{HF}$, $22 \pm 5 \mathrm{bpm}$; controls, $24 \pm 4 \mathrm{bpm}$ ), perceived effort (HF, $8 \pm 1$ units; controls, $8 \pm 1$ units), and systolic blood pressure (HF, $22 \pm 2 \mathrm{mmHg}$; controls, $23 \pm 1 \mathrm{mmHg}$ ) were similar between groups $(\mathrm{P}>0.05) . \quad \mathrm{SpO}_{2}$ was lower $(\mathrm{P}<0.05)$ in patients with HF [pre, 96\% (94-97\%); fatigue, $95 \%(95-96 \%)$ ] than in healthy controls [pre, 97\% (96$98 \%)$; fatigue, $97 \%(96-98 \%)$ ], but did not change during the protocol $(P>0.05)$. Figure 2 shows that at respiratory fatigue, blood volume (left panels) and tissue oxygenation (right panels) decreased in both intercostal (upper panels) and forearm muscles (lower panels) only in patients with HF $(\mathrm{P}<0.05)$.

\section{Discussion}

To our knowledge, this is the first study to investigate the triggering mechanism of the respiratory metaboreflex response during respiratory muscle fatigue in patients with $\mathrm{HF}$. We tested the hypothesis that there would be a reduction in intercostal muscle oxygenation during respiratory fatigue with onset of the metaboreflex response in 

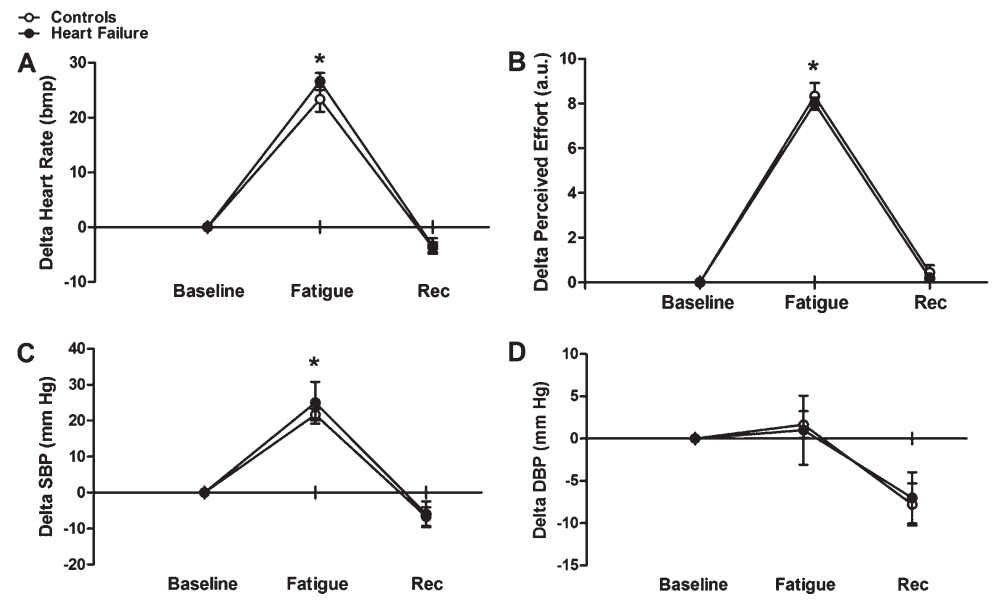

Figure 1. Changes in heart rate $(A)$, perceived effort $(B)$, systolic blood pressure (SBP) $(C)$, and diastolic blood pressure (DBP) $(D)$ during the respiratory fatigue protocol in patients with heart failure and in healthy controls. Data are reported as means \pm SD. Fatigue: average of the last $3 \mathrm{~s}$ before fatigue occurred; REC: recovery. ${ }^{*} \mathrm{P}<0.05$ fatigue $v$ s baseline (two-way ANOVA followed by the Bonferroni post hoc test). patients with HF. Our results showed a significant reduction in intercostal and forearm muscle oxygen saturation from rest to fatigue in the HF group, which was not observed in the healthy group. It can be inferred from these results that the increased work of the respiratory muscles led to fatigue and the accumulation of local metabolites, which triggered a metaboreflex response generating a reflex peripheral vasoconstriction response.

Reduced exercise capacity is a major feature in individuals with HF (1) and a powerful predictor of mortality $(2,10)$. The mechanisms involved in this impaired ability to perform exercise encompass both central and peripheral mechanisms, such as blunted increase in cardiac output, increased sympathetic drive (11), altered distribution of blood flow, skeletal muscle atrophy, and metabolic changes $(12,13)$. In addition, respiratory dysfunction has been recognized as an integral part of the HF syndrome due to increase in capillary wedge pressure, leading to impaired alveolar gas exchange. Accordingly, respiratory dysfunction has been shown to represent an independent risk factor for overall mortality (2). The mechanisms responsible for fatigue and dyspnea in HF patients are multifactorial and include intrinsic respiratory muscle fiber abnormalities $(14,15)$. Typical histological changes include muscle atrophy, changes in myofibrillar protein composition with increase in the proportion of type II fatigable fibers, decreases in oxidative enzymes (type I fibers), and reductions in mitochondrial size and number $(15,16)$.

In addition to these established respiratory mechanisms in HF, Dempsey et al. $(3,4)$ proposed that respiratory metaboreflex plays a major role in contributing to the reflex increase in sympathetic activity that is triggered by the buildup of metabolites within respiratory muscle fibers produced during contraction. This is analogous to the so-called exercise pressor reflex, where the accumulation of metabolites in appendicular skeletal muscles increases afferent signals through sensitive type III and IV fibers and augments reflex sympathetic drive (5). The experimental evidence for a respiratory metaboreflex includes the observation in anesthetized rats that fatigue of the diaphragm increases the firing rate of type IV afferent metaboreceptors from this muscle, while the activity of type III mechanoreceptors is not
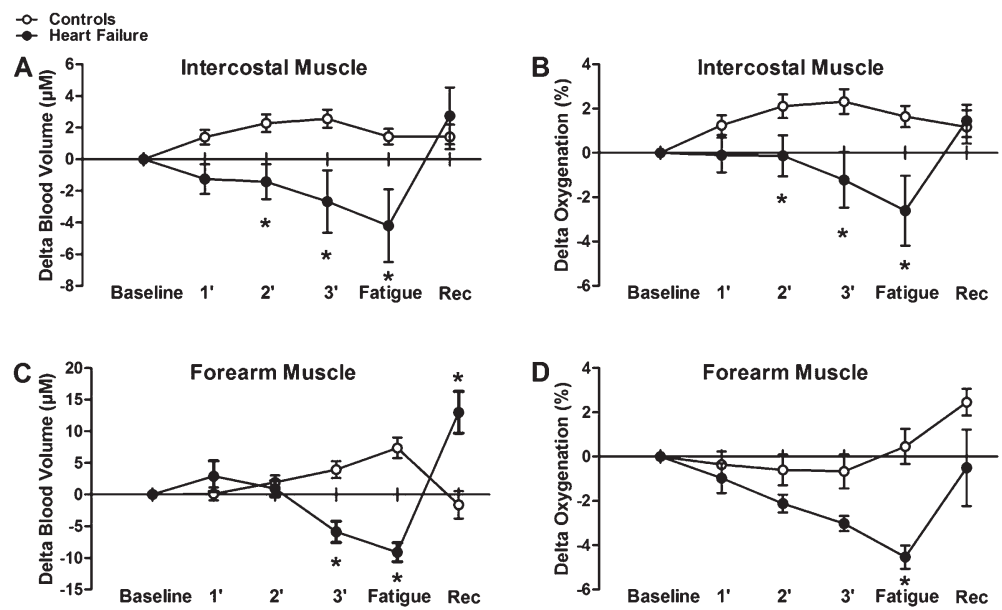

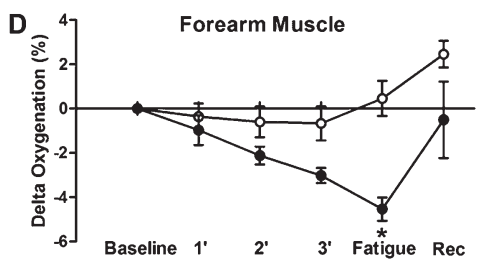

Figure 2. Changes in tissue blood volume ( $A$ and $C)$ and oxygenation $(B$ and $D)$ at intercostal and forearm muscles during respiratory muscle fatigue in patients with heart failure and in healthy controls. Data are reported as means \pm SD. Fatigue: average of the last $3 \mathrm{~s}$ before fatigue occurred; REC: recovery. ${ }^{*} \mathrm{P}<0.05$ fatigue vs baseline (two-way ANOVA followed by the Bonferroni post hoc test). 
altered (8). In healthy humans, respiratory exercise with a load equivalent to $60 \%$ of maximum inspiratory and expiratory pressures increased sympathetic nerve discharge (directly measured in the peroneal nerve), and reduced blood flow to the limb at rest $(17,18)$. Similar effects have also been shown in patients with $\operatorname{HF}(7,19,20)$.

The present results showing reduced intercostal and forearm perfusion and tissue oxygenation in patients with HF could be explained by mechanisms other than the activation of the respiratory metaboreflex operating during the respiratory fatigue protocol, such as reduction of overall tissue perfusion and decrease in $\mathrm{SpO}_{2}$. By definition, patients with HF have systolic dysfunction (decreased left ventricular ejection fraction at rest) and thus limited capacity to increase systemic blood flow during exercise. However, the similar increase in blood pressure during the respiratory fatigue protocol in both groups suggests that patients with HF had preserved capacity to increase systemic blood flow at this level of effort. Another potential mechanism to explain the present results would be a decrease in arterial oxygen saturation during the protocol, leading to reduced tissue oxygenation to intercostal and forearm muscles. Although $\mathrm{SpO}_{2}$ was lower in patients with $\mathrm{HF}$, it did not change during the respiratory protocol and thus cannot explain the differences appearing during this period. Altogether, the present results do not seem to be caused by a systemic effect on either blood flow or arterial oxygenation, but rather a local mechanism in intercostal muscle leading to decreased local muscle deoxygenation triggering a respiratory metaboreflex.

\section{References}

1. Piepoli M, Chua TP, Coats AJ. Exercise intolerance in patients with chronic heart failure. Eur Heart $J$ 1995; 16 : 1744-1745.

2. Meyer FJ, Borst MM, Zugck C, Kirschke A, Schellberg D, Kubler W, et al. Respiratory muscle dysfunction in congestive heart failure: clinical correlation and prognostic significance. Circulation 2001; 103: 2153-2158, doi: 10.1161/01.CIR.103. 17.2153.

3. Mancini DM, Ferraro N, Nazzaro D, Chance B, Wilson JR. Respiratory muscle deoxygenation during exercise in patients with heart failure demonstrated with near-infrared spectroscopy. J Am Coll Cardiol 1991; 18: 492-498, doi: 10.1016/0735-1097(91)90605-9.

4. Rodman JR, Henderson KS, Smith CA, Dempsey JA. Cardiovascular effects of the respiratory muscle metaboreflexes in dogs: rest and exercise. J Appl Physiol 2003; 95: 1159-1169.

5. Murphy MN, Mizuno M, Mitchell JH, Smith SA. Cardiovascular regulation by skeletal muscle reflexes in health and disease. Am J Physiol Heart Circ Physiol 2011; 301: H1191-H1204, doi: 10.1152/ajpheart.00208.2011.

6. Dempsey JA, Romer L, Rodman J, Miller J, Smith C. Consequences of exercise-induced respiratory muscle work. Respir Physiol Neurobiol 2006; 151: 242-250, doi: 10.1016/
A similar increase in blood pressure during respiratory fatigue has been described previously in patients with HF $(7,18)$. Intercostal and forearm muscles comprise a small mass and thus a very small portion of the systemic arterial circulation. Therefore, it is most likely that the increase in blood pressure during respiratory fatigue was caused by similar elevations in cardiac output in the face of unchanged systemic vascular resistance in both groups. This suggests that the observed changes in intercostal muscle were not a systemic but a local effect of respiratory effort.

Near-infrared spectroscopy has been used as a tool for assessment of muscle oxygenation and validated as a reliable, noninvasive method for analysis of blood volume in the intercostal muscles of healthy subjects (17). Vogiatzis et al. (9) used the same method to study oxygenation in intercostal and vastus lateralis muscles in elite athletes during exercise. They observed that maximum exercise of the lower limbs reduced flow to the intercostal muscles, an effect not seen during equivalent hyperpnea at rest $(9,17)$.

The present results provide original evidence that the metaboreflex during respiratory muscle fatigue in HF is triggered by an oxygen demand/delivery mismatch in respiratory muscles leading to a reflex reduction of perfusion of peripheral limb muscles.

\section{Acknowledgments}

Research supported by CNPq, FINEP, CAPES, and FAPERJ.

j.resp.2005.12.015.

7. Borghi-Silva A, Carrascosa C, Oliveira CC, Barroco AC, Berton DC, Vilaca D, et al. Effects of respiratory muscle unloading on leg muscle oxygenation and blood volume during high-intensity exercise in chronic heart failure. $A m ~ J$ Physiol Heart Circ Physiol 2008; 294: H2465-H2472, doi: 10.1152/ajpheart.91520.2007.

8. Hill JM. Discharge of group IV phrenic afferent fibers increases during diaphragmatic fatigue. Brain Res 2000; 856: 240-244, doi: 10.1016/S0006-8993(99)02366-5.

9. Vogiatzis I, Athanasopoulos D, Habazettl H, Kuebler WM, Wagner $\mathrm{H}$, Roussos $\mathrm{C}$, et al. Intercostal muscle blood flow limitation in athletes during maximal exercise. J Physiol 2009; 587: 3665-3677, doi: 10.1113/jphysiol.2009.171694.

10. Hambrecht R, Gielen S, Linke A, Fiehn E, Yu J, Walther C, et al. Effects of exercise training on left ventricular function and peripheral resistance in patients with chronic heart failure: A randomized trial. JAMA 2000; 283: 3095-3101, doi: 10.1001/jama.283.23.3095.

11. Barretto AC, Santos AC, Munhoz R, Rondon MU, Franco FG, Trombetta IC, et al. Increased muscle sympathetic nerve activity predicts mortality in heart failure patients. Int $\mathrm{J}$ Cardiol 2009; 135: 302-307, doi: 10.1016/j.ijcard.2008.03.056.

12. Myers J, Gianrossi R, Schwitter J, Wagner D, Dubach P. 
Effect of exercise training on postexercise oxygen uptake kinetics in patients with reduced ventricular function. Chest 2001; 120: 1206-1211, doi: 10.1378/chest.120.4.1206.

13. Negrao CE, Middlekauff HR. Exercise training in heart failure: reduction in angiotensin II, sympathetic nerve activity, and baroreflex control. J Appl Physiol 2008; 104 : 577-578, doi: 10.1152/japplphysiol.01368.2007

14. Lindsay DC, Lovegrove CA, Dunn MJ, Bennett JG, Pepper JR, Yacoub $\mathrm{MH}$, et al. Histological abnormalities of muscle from limb, thorax and diaphragm in chronic heart failure. Eur Heart J 1996; 17: 1239-1250, doi: 10.1093/oxfordjournals.eurheartj. a015042.

15. Lecarpentier Y, Pery N, Coirault C, Scalbert E, Desche P, Suard I, et al. Intrinsic alterations of diaphragm muscle in experimental cardiomyopathy. Am Heart J 1993; 126: 770776, doi: 10.1016/0002-8703(93)90928-3.

16. Tikunov BA, Mancini D, Levine S. Changes in myofibrillar protein composition of human diaphragm elicited by congestive heart failure. J Mol Cell Cardiol 1996; 28: 2537-2541, doi: 10.1006/jmcc.1996.0245.

17. Guenette JA, Vogiatzis I, Zakynthinos S, Athanasopoulos D, Koskolou M, Golemati S, et al. Human respiratory muscle blood flow measured by near-infrared spectroscopy and indocyanine green. J Appl Physiol 2008; 104: 1202-1210, doi: 10.1152/japplphysiol.01160.2007

18. Derchak PA, Sheel AW, Morgan BJ, Dempsey JA. Effects of expiratory muscle work on muscle sympathetic nerve activity. J Appl Physiol 2002; 92: 1539-1552.

19. Dall'Ago P, Chiappa GR, Guths H, Stein R, Ribeiro JP. Inspiratory muscle training in patients with heart failure and inspiratory muscle weakness: a randomized trial. J Am Coll Cardiol 2006; 47: 757-763, doi: 10.1016/j.jacc.2005.09. 052.

20. Chiappa GR, Roseguini BT, Vieira PJ, Alves CN, Tavares A, Winkelmann ER, et al. Inspiratory muscle training improves blood flow to resting and exercising limbs in patients with chronic heart failure. J Am Coll Cardiol 2008; 51: 16631671, doi: 10.1016/j.jacc.2007.12.045. 